\title{
NUMERICAL PROJECTION METHOD \\ FOR INVERSE FOURIER TRANSFORM \\ AND ITS APPLICATION
}

\author{
Andrey S. Krylov and Anton V. Liakishev \\ Faculty of Computational Mathematics and Cybernetics, \\ Moscow State University, Vorobievy Gory, 119899 Moscow, Russia \\ e-mail:kryl@cs.msu.su
}

\begin{abstract}
Numerical projection method of the Fourier transform inversion from data given on a finite interval is proposed. It is based on an expansion of the solution into a series of eigenfunctions of the Fourier transform. The number of terms of the expansion depends on the length of the data interval. Convergence of the solution of the method is proved. The projection method for the case of the sine Fourier transform and the set of the odd Hermite functions being its eigenfunctions are examined and applied to numerical Fourier filtering.

Keywords: Fourier transform, Hermite functions, Fourier filtering, projection method, convergence theorem, numerical method.
\end{abstract}

AMS Classification (1991) : 42A38, 65R10, 33C45. 


\section{Introduction}

The Hermite functions as the eigenfunctions of the Fourier transform $[1,2,3]$ are widely used in the Fourier analysis. Expansions into series of the Hermite functions in Fourier analysis applications can be found in image coding and analysis [4, 5], statistics [6], electrophysiology [7], Monte-Carlo computations [8], diffraction structure investigations $[9,10]$ and other areas of sciences.

The Hermite functions have also attracted considerable interest in applications as real functions minimizing the Gabor uncertainty $[11,12]$ for the Fourier transform. The Gabor principle by analogy with the Heisenberg uncertainty principle is that the product of localization of a function in real (time) and re-

ciprocal (frequency) spaces has a lower bound and the Gabor functions [13] are complex functions realizing this bound.

In many cases we know that the data to be processed by the Fourier transform are not compact supported. Nevertheless, they are given on a finite interval only. The principal problem in this situation is to find a criterion to estimate the number of terms to be used for the expansion of the data into a series of the Hermite functions. The idea to relate the number of terms with the length of the experimental interval and thus to take into consideration the additional physical information on the data was first introduced in [14]. The aim of our paper is to justify this approach and to show some application of the method to the problem of the Fourier filtering.

The outline of the paper is the following. We consider in $\S 2$ a projection method of solution of an inverse problem for linear operator equations including as a special case the problem of the Fourier transform inversion with the data given on a finite interval. A theorem of convergence of the solution for the method is proved. The properties of the Hermite functions are investigated in $\S 3$. The feasibility of the proposed method for inversion of the sine Fourier transform using approximation of solution by a series of the Hermite functions is shown. The application of the method of Fourier transform inversion in the Fourier filtering is illustrated in $\S 4$. The practical advantages of the proposed 
projection method are discussed in $\S 5$.

\section{Preliminaries}

We consider the problem of finding of a function $z$ from known $u$

$$
A z=u, \quad A: L_{2}[0, \infty) \rightarrow L_{2}[0, \infty)
$$

where $\mathrm{A}$ is a linear continuous operator and the operator $A$ has a complete orthonormal in $L_{2}[0, \infty)$ system of eigenfunctions $\left\{\varphi_{i}\right\}, i=1,2, \ldots$ such that the absolute values of the eigenvalues $\left\{\lambda_{i}\right\}$, are bounded and are bounded away from zero:

$$
\infty>\Lambda>\left|\lambda_{i}\right|>\lambda>0 .
$$

The inverse problem (1) is well-posed [15] and has an unique solution $z$ for the known $u$. If instead of the exact right side $u(x)$ of the equation (1) we have a function $u^{\delta}(x)$ and an error $\delta$

$$
\left\|u^{\delta}-u\right\|_{L_{2}[0, \infty)} \leq \delta
$$

then $\left\|z^{\delta}-z\right\|_{L_{2}[0, \infty)} \underset{\delta \rightarrow 0}{\longrightarrow} 0$. Here $z^{\delta}$ is the solution of the equation $A z^{\delta}=u^{\delta}$.

In many cases the information on $u(x)$ can be obtained on a finite interval $\left[0, x_{\max }\right]$ only and the problem can be posed as

$$
A z_{x_{\max }}=u_{x_{\max }}, \quad A: L_{2}[0, \infty) \rightarrow L_{2}\left[0, x_{\max }\right] .
$$

In general, we have not the uniqueness of the solution $z_{x_{\max }}$ for this problem and thus the problem is ill-posed.

Consider now the case when instead of $u_{x_{\max }}(x)$ we have $u^{\delta}(x)$ on the interval $\left[0, x_{\max }\right]$. In this situation it is reasonable to find an approximation of the unique solution of the well-posed problem (1) instead of finding of an approximation of one of the solutions of the problem (4). The lack of the information for $x>x_{\max }$ can be considered as an "error" in the right side of the equation (1). So, it is necessary to find an approximate solution $z_{x_{\max }}^{\delta}$ that $\left\|z_{x_{\max }}^{\delta}-z\right\|_{\substack{x_{\max } \rightarrow \infty \\ \delta \rightarrow 0}}^{\longrightarrow} 0$, where $z$ is the solution of (1). 
Definition. Set of functions $\left\{\psi_{i}\right\}$ is called norm-ordered with index $k$ if

$$
\left\|\psi_{i}\right\|_{L_{2}[x, \infty)}<\left\|\psi_{i+j}\right\|_{L_{2}[x, \infty)}, \quad i=1,2, \ldots, \quad j=k, k+1, \ldots,
$$

for all $x$, where $x>x_{1} \geq 0$ and $k \geq 1$ is a constant.

If for some $k>0, \infty>x_{1} \geq 0$ we can reindex set of the eigenfunctions of our operator $A$ so that the set $\varphi_{i}$ becomes norm-ordered with index $k$, then the algorithm of solution of our problem can be based on the following theorem.

Theorem 1. Let

$$
z_{x_{\text {max }}}^{\delta}(y)=\sum_{i=1}^{n_{m}} d_{x_{\text {max }}}^{\delta, i} \varphi_{i}(y)
$$

where

$$
d_{x_{\text {max }}}^{\delta, i}=\frac{c_{x_{\text {max }}}^{\delta, i}}{\lambda_{i}}, \quad c_{x_{\text {max }}}^{\delta, i}=\int_{0}^{x_{\max }} u_{x_{\max }}^{\delta}(x) \varphi_{i}(x) d x, \quad i=1,2, \ldots, n_{m},
$$

and $n_{m}$ is the maximum $n$, satisfying the condition

$$
\left\|\varphi_{i}\right\|_{L_{2}\left[x_{\max }, \infty\right)} \leq \frac{\varepsilon}{n^{1 / 2+\sigma}}, \quad i=1,2, \ldots, n
$$

$\varepsilon>0, \sigma>0-$ constants. Then

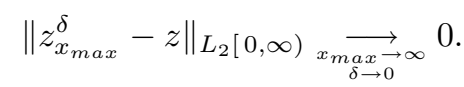

\section{Proof.}

The second term in the right side of the inequality

$$
\left\|z_{x_{\max }}^{\delta}-z\right\|_{L_{2}[0, \infty)} \leq\left\|z_{x_{\max }}^{\delta}-z^{\delta}\right\|_{L_{2}[0, \infty)}+\left\|z^{\delta}-z\right\|_{L_{2}[0, \infty)}
$$

tends to zero for $\delta \rightarrow 0$. For the first term the following estimate has place

$$
\begin{gathered}
\left\|z_{x_{\max }}^{\delta}-z_{\delta}\right\|_{L_{2}[0, \infty)}=\left(\int_{0}^{\infty}\left(z_{x_{\max }}^{\delta}(y)-z_{\delta}(y)\right)^{2} d y\right)^{1 / 2} \leq \\
\leq\left[\int_{0}^{\infty}\left(\sum_{i=1}^{n_{m}}\left(c_{x_{\max }}^{\delta, i}-c_{i}^{\delta}\right) \frac{1}{\lambda_{i}} \varphi_{i}(y)\right)^{2} d y\right]^{1 / 2}+\left[\int_{0}^{\infty}\left(\sum_{i=n_{m}+1}^{\infty} c_{i}^{\delta} \frac{1}{\lambda_{i}} \varphi_{i}(y)\right)^{2} d y\right]^{1 / 2} .
\end{gathered}
$$


According to (5),(6), $n_{m} \rightarrow \infty$ when $x_{\max } \rightarrow \infty$. Therefore the second term tends to zero when $x_{\max } \rightarrow \infty$.

At the same time,

$$
\begin{aligned}
& {\left[\int_{0}^{\infty}\left(\sum_{i=1}^{n_{m}}\left(c_{x_{\max }}^{\delta, i}-c_{i}^{\delta}\right) \frac{1}{\lambda_{i}} \varphi_{i}(y)\right)^{2} d y\right]^{1 / 2}=} \\
& =\left[\int_{0}^{\infty}\left(\sum_{i=1}^{n_{m}}\left(\int_{0}^{x_{\max }} u^{\delta}(\xi) \varphi_{i}(\xi) d \xi-\int_{0}^{\infty} u^{\delta}(\xi) \varphi_{i}(\xi) d \xi\right) \frac{1}{\lambda_{i}} \varphi_{i}(y)\right)^{2} d y\right]^{1 / 2}= \\
& =\left[\int_{0}^{\infty}\left(\sum_{i=1}^{n_{m}} \int_{x_{\max }}^{\infty} u^{\delta}(\xi) \varphi_{i}(\xi) d \xi \frac{1}{\lambda_{i}} \varphi_{i}(y)\right)^{2} d y\right]^{1 / 2} \leq \\
& \leq \frac{1}{\lambda}\left[\int_{0}^{\infty}\left(\sum_{i=1}^{n_{m}}\left(\int_{c_{\max }}^{\infty} u^{\delta^{2}}(\xi) d \xi\right)^{1 / 2}\left(\int_{c_{\max }}^{\infty} \varphi_{i}^{2}(\xi) d \xi\right)^{1 / 2} \varphi_{i}(y)\right)^{2} d y\right]^{1 / 2} \leq \\
& \leq \frac{1}{\lambda}\left\|u^{\delta}\right\| \frac{\varepsilon}{n_{m}^{1 / 2+\sigma}}\left(\int_{0}^{\infty}\left(\sum_{i=1}^{n_{m}} \varphi_{i}(y)\right)^{2} d y\right)^{1 / 2} \leq \frac{\left\|u^{\delta}\right\| \varepsilon}{\lambda n_{m}^{\sigma}} \underset{n_{m} \rightarrow \infty}{\longrightarrow} 0,
\end{aligned}
$$

Q.E.D.

Theorem 2. For the coefficients $d_{x_{\max }, i}^{\delta}, \quad i=1,2, \ldots, n_{m}, \quad$ the following estimate has place

$$
\left|d_{x_{\max }}^{\delta, i}-d_{i}\right| \leq \delta+\|u\|_{L_{2}\left[x_{\max }, \infty\right)} \frac{\varepsilon}{n_{m}^{1 / 2+\sigma}},
$$

where

$$
d_{i}=\frac{1}{\lambda_{i}} \int_{0}^{\infty} u(x) \varphi_{i}(x) d x
$$

\section{Proof.}

$$
\begin{gathered}
\left|d_{x_{\text {max }}}^{\delta, i}-d_{i}\right|=\left|\int_{0}^{x_{\max }}\left(u(x)-u^{\delta}(x)\right) \varphi_{i}(x) d x+\int_{x_{\max }}^{\infty} u(x) \varphi_{i}(x) d x\right| \leq \\
\leq\left\|u(x)-u^{\delta}(x)\right\|_{L_{2}\left[0, x_{\max }\right]}\left\|\varphi_{i}(x)\right\|_{L_{2}\left[0, x_{\max }\right]}+\|u(x)\|_{L_{2}\left[x_{\max }, \infty\right)}\left\|\varphi_{i}(x)\right\|_{L_{2}\left[x_{\max }, \infty\right)} \leq \\
\leq \delta+\|u(x)\|_{L_{2}\left[x_{\max }, \infty\right)} \frac{\varepsilon}{n_{m}^{1 / 2+\sigma}},
\end{gathered}
$$

Q.E.D. 


\section{Hermite functions}

The Hermite functions are the eigenfunction of the Fourier transform [2]. The properties of this set of functions enables us to construct an algorithm of inversion of the Fourier transform described above in the general case.

The Hermite polynomials are defined as $[16,17]$

$$
H_{n}(x)=(-1)^{n} e^{x^{2}} \frac{d^{n} e^{-x^{2}}}{d x^{n}},
$$

and satisfy the following relations

$$
\begin{gathered}
H_{n}^{\prime}(x)=2 n H_{n-1}(x), \\
H_{n+1}(x)=2 x H_{n}(x)-2 n H_{n-1}(x) .
\end{gathered}
$$

The Hermite functions are defined as follows

$$
\Phi_{n}(x)=e^{-\frac{x^{2}}{2}} H_{n}(x) .
$$

They satisfy the recursion relation

$$
\Phi_{n+1}(x)=2 x \Phi_{n}(x)-2 n \Phi_{n-1}(x) .
$$

The same definitions and relations for the orthonormal in $L_{2}[0, \infty)$ Hermite polynomials and functions are

$$
\begin{gathered}
\hat{H}_{n}(x)=\frac{(-1)^{n}}{\sqrt{2^{n-1} n ! \sqrt{\pi}}} e^{x^{2}} \frac{d^{n} e^{-x^{2}}}{d x^{n}}, \\
\hat{H}_{n+1}(x)=x \sqrt{\frac{2}{n+1}} \hat{H}_{n}(x)-\sqrt{\frac{n}{n+1}} \hat{H}_{n-1}(x), \\
\hat{\Phi}_{n}(x)=e^{-\frac{x^{2}}{2}} \hat{H}_{n}(x)=\frac{1}{\sqrt{2^{n-1} n ! \sqrt{\pi}}} e^{-\frac{x^{2}}{2}} H_{n}(x), \quad\left\|\hat{\Phi}_{n}\right\|_{L_{2}[0, \infty)}=1, \\
\hat{\Phi}_{n+1}(x)=x \sqrt{\frac{2}{n+1}} \hat{\Phi}_{n}(x)-\sqrt{\frac{n}{n+1}} \hat{\Phi}_{n-1}(x) .
\end{gathered}
$$


Lemma 1. The norms of the Hermite functions $\Phi_{n}$ satisfy the recursion relation

$$
\left\|\Phi_{n}\right\|_{L_{2}[0, R]}^{2}=2 n\left\|\Phi_{n-1}\right\|_{L_{2}[0, R]}^{2}-\Phi_{n}(R) \Phi_{n-1}(R) .
$$

\section{Proof.}

Applying (8) and integrating by parts we obtain

$$
\begin{gathered}
\left\|\Phi_{n}\right\|_{L_{2}[0, R]}^{2}=\int_{0}^{R} e^{-x^{2}} H_{n}(x) H_{n}(x) d x=\frac{1}{2(n+1)} \int_{0}^{R} e^{-x^{2}} H_{n}(x) d H_{n+1}(x)= \\
=\frac{1}{2(n+1)}\left[\left.e^{-x^{2}} H_{n}(x) H_{n+1}(x)\right|_{0} ^{R}-\int_{0}^{R} H_{n+1}(x) d\left(e^{-x^{2}} H_{n}(x)\right)\right]= \\
=\frac{1}{2(n+1)} e^{-R^{2}} H_{n}(r) H_{n+1}(R)- \\
-\frac{1}{2(n+1)} \int_{0}^{R} H_{n+1}(x)\left(-2 x e^{-x^{2}} H_{n}(x) d x+e^{-x^{2}} d H_{n}(x)\right) .
\end{gathered}
$$

According to (9) we have

$$
\begin{gathered}
\int_{0}^{R} 2 x H_{n}(x) H_{n+1}(x) e^{-x^{2}} d x=\int_{0}^{R} H_{n+1}^{2}(x) e^{-x^{2}} d x+\int_{0}^{R} 2 n H_{n-1}(x) H_{n+1}(x) e^{-x^{2}} d x= \\
=\int_{0}^{R} H_{n+1}^{2}(x) e^{-x^{2}} d x+\int_{0}^{R} H_{n+1}(x) e^{-x^{2}} d H_{n}(x) .
\end{gathered}
$$

Therefore

$$
\left\|\Phi_{n}\right\|_{L_{2}[0, R]}^{2}=\frac{1}{2(n+1)} e^{-R^{2}} H_{n}(R) H_{n+1}(R)+\frac{1}{2(n+1)} \int_{0}^{R} H_{n+1}^{2}(x) e^{-x^{2}} d x .
$$

Thus, for the Hermite functions we have

$$
\left\|\Phi_{n}\right\|_{L_{2}[0, R]}^{2}=\frac{1}{2(n+1)}\left(\Phi_{n}(r) \Phi_{n+1}(R)+\left\|\Phi_{n+1}\right\|_{L_{2}[0, R]}^{2}\right),
$$

and the required result follows. 
For the orthonormal Hermite functions $\hat{\Phi}_{n}$ the recursion relation may be written as follows

$$
\left\|\hat{\Phi}_{n}\right\|_{L_{2}[0, R]}^{2}=\left\|\hat{\Phi}_{n-1}\right\|_{L_{2}[0, R]}^{2}-\frac{1}{\sqrt{2 n}} \hat{\Phi}_{n}(R) \hat{\Phi}_{n-1}(R) .
$$

We will show the idea of the method of the Fourier transform inversion with the case of the sine Fourier transform on a half-line.

The odd Hermite functions (see Fig. 1)

$$
\Psi_{n}(x)=\hat{\Phi}_{2 n+1}(x)
$$

are the eigenfunctions of the sine Fourier transform

$$
\int_{0}^{\infty} \Psi_{n}(x) \sin x y d x=(-1)^{n} \sqrt{\frac{\pi}{2}} \Psi_{n}(y) .
$$

They satisfy the following recursion relation

$$
\Psi_{n+1}(x)=\frac{2 x^{2}-4 n-3}{\sqrt{(2 n+2)(2 n+3)}} \Psi_{n}(x)-\sqrt{\frac{2 n(2 n+1)}{(2 n+2)(2 n+3)}} \Psi_{n-1}(x) .
$$

Lemma 1a. The norms of the orthonormal Hermite functions $\Psi_{n}$ satisfy the recursion relation

$$
\begin{gathered}
\left\|\Psi_{n}\right\|_{L_{2}[0, R]}^{2}=\left\|\Psi_{n-1}\right\|_{L_{2}[0, R]}^{2}- \\
-\frac{1}{2 R}\left(\Psi_{n}^{2}(R)+\frac{4 n+1}{\sqrt{2 n(2 n+1)}} \Psi_{n}(R) \Psi_{n-1}(R)+\Psi_{n-1}^{2}(R)\right) .
\end{gathered}
$$

The general behavior of the norms $\left\|\Psi_{n}\right\|_{L_{2}[0, x]}^{2}$ as functions of $x$ is shown in Fig. 2. According to (21) there are intersections between $\left\|\Psi_{n}\right\|_{L_{2}[0, x]}^{2}$ and $\left\|\Psi_{n+1}\right\|_{L_{2}[0, x]}^{2}$. Nevertheless, the following theorem has place [18].

Theorem 3. The set of functions $\left\{\Psi_{n}\right\}$ is norm-ordered with index 2 for $x_{1}=1$, that is

$$
\left\|\Psi_{n+i}\right\|_{L_{2}[x, \infty)}^{2}>\left\|\Psi_{n-1}\right\|_{L_{2}[x, \infty)}^{2}, \quad \forall i, n \geq 1, \forall x \geq 1
$$




\section{Projection scheme of Fourier filtering}

The general scheme of the Fourier filtering for function smoothing looks as follows

$$
f(x) \stackrel{F}{\longrightarrow} \hat{f}(k) \longrightarrow \breve{f}(k)=\hat{f}(k) \cdot G(k) \stackrel{F^{-1}}{\longrightarrow} \tilde{f}(x),
$$

where $F$ is the Fourier transform and $G(k)$ is a "window" function. We will consider the case of the sine Fourier filtering

$$
F(f(x))=F_{s}(f(x))=\sqrt{\frac{2}{\pi}} \int_{0}^{\infty} f(x) \sin x k d x .
$$

An example of a window function is the "natural" window: $G_{0}(k)=1$ for $0<k \leq k_{\max }$, and $G_{0}(k)=0$ for $k>k_{\max }$. Its use blur the initial function $f(x), x \geq 0$ and add false oscillations to the result. These disadvatages are typical for all window functions used in practice [19].

The projection method enables us to construct an effective algorithm of the Fourier filtering to avoid these problems and to store the filtered function in a compact form.

Instead of the scheme (23) the filtering is performed by approximating of $\hat{f}(k)$ with a finite series of the odd Hermite functions $\Psi_{n}$ concentrated on $\left[0, k_{\max }\right]$

$$
\begin{gathered}
\tilde{f}(x)=\sum_{i=0}^{n_{\mathrm{m}}} c_{i} \Psi_{i}(x), \quad c_{i}=(-1)^{i} \int_{0}^{k_{\max }} \hat{f}_{0}(k) \Psi_{i}(k) d k, \\
n_{\mathrm{m}}=\max \left\{n \mid\left\|\Psi_{i}\right\|_{L_{2}\left[0, k_{\max }\right]}^{2} \geq 1-\varepsilon\right\},
\end{gathered}
$$

where $1>\varepsilon>0$ is a constant.

By this method the multiplication of $\hat{f}(k)$ by a window function $G(k)$ in the scheme (23) is replaced by expanding of $\hat{f}(k)$ into the finite series of $n_{\mathrm{m}}$ odd Hermite functions concentrated on the selected interval. The $n_{\mathrm{m}}$ value can be numerically found using Lemma 1a.

Figs. 3-5 show an example of use of the projection method in the Fourier filtering. We added uniformly distributed error to the initial function $f(x)$. The resulting function $f_{\delta}(x)$ was treated by the Fourier filtering with the natural 
window and with the projection method for $k_{\max }=8$. Thus, the sine Fourier transform $\hat{f}(k)$ of $f_{\delta}(x)$ was cut at $k=8$ (see Fig. 4 ) and was continued by 0 for $k>k_{\max }$. Then the inverse sine Fourier transform was calculated by the projection method (the obtained approximation $\hat{f}_{p}(k)$ with $\varepsilon=0.1, n_{\mathrm{m}}=17$ is plotted with dotted line in Fig. 4) and by the explicit formula of the sine Fourier transform inversion. The results by the projection method $\tilde{f}_{p}(x)$ and by the natural window application $\tilde{f}_{s}(x)$ are shown in Fig. 3 along with initial functions $f(x)$ and $f_{\delta}(x)$. Figs. 5a, 5b show more detailed parts of Fig. 3.

\section{Conclusion}

The methods usually used for numerical sine Fourier transform inversion from data given on a finite interval are based either on the explicit formula for the inverse Fourier transform or on the solution of an integral equation of the first kind with a fixed upper limit of integration.

The standard method based on the use of the explicit formula of the sine Fourier transform is equivalent to the expansion of the data into an infinite series of the odd Hermite functions with the assumption that the data is zero outside the experimental interval. In this case the use of the Hermite functions which are not concentrated on the experimental interval in practice adds noise to the result, because the coefficients of the expansion for these functions depend critically on the unavailable data outside our interval.

The inverse problem for the integral equation of the first kind being an illposed problem is solved, for example, in [20], using the regularizing method of Tikhonov [15]. The idea to replace the initial problem which has a continuos dependence of the result on the initial data, to an unstable problem and to suppress then this unstability by a regularizing method does not look natural. It is preferable to use methods, which take into account the properties of the sine Fourier transform while no important information about the initial problem is rejected.

The projection method was illustrated for the case of the sine Fourier trans- 
form inversion. This method can be also used for the complex Fourier transform [21]. The projection method in the case of the Hankel transform [22] is based on the system of Laguerre functions.

Use of Gauss-Hermite quadrature to find the coefficients of the expansion [3] allows us to construct fast algorithms for the proposed method.

Finally, some of the features of the projection method can be mentioned: (i) Additional physical information is used to find the solution (length of the data interval), (ii) Smoothing of the result is performed without loss of the physical information, (iii) It is possible to test if the data quality is sufficient for the given length of the data interval, basing on the quality of the fitting of the data obtained, (iv) The approximation of the data and its Fourier transform are stored in a compact form.

The authors are grateful to Professor A.M. Denisov for many valuable discussions and helpful suggestions.

\section{REFERENCES}

[1] N. Wiener, The Fourier Integral and Certain of its Applications, Cambridge, 1933.

[2] E.C. Titchmarsh, Introduction to the Theory of Fourier Integrals, Oxford University Press, N.Y., 1937.

[3] W.F. Eberlein, A New Method for Numerical Evaluation of the Fourier Transform, J. of Math. Analysis and Appl., 65, 1978, 80-84.

[4] J.-B. Martens, The Hermite Transform - Theory, IEEE Trans. Acoust., Speech, Signal Processing, 38, 1990, 1595-1606.

[5] J.-B. Martens, The Hermite Transform - Applications, IEEE Trans. Acoust., Speech, Signal Processing, 38, 1990, 1607-1618. 
[6] R. Deutsch, Nonlinear Transformations of Random Processes, Prentice-Hall Inc., Englewood Cliffs, N.J., 1962.

[7] L.R. Lo Conte, R. Merletti and G.V. Sandri, Hermite Expansions of Compact Support Waveforms: Applications to Myoelectric Signals, IEEE Trans. Biomed. Eng., 41, 1994, 1147-1159.

[8] A.J. Chorin, Hermite Expansions in Monte-Carlo Computation, J. Comp. Physics, 8, 1971, 472-482.

[9] A.S. Krylov and B.M. Shchedrin, Numerical Method for Radial Distribution Function Calculation, Crystallographya, 34, 1989, 1088-1092 (in Russian).

[10] D.I. Svergun, A Direct Indirect Method of Small-Angle Scattering Data Treatment, J. Appl. Cryst., 26, 1993, 258-267.

[11] D. Gabor, Theory of Communication, J. Inst. Electr. Eng., 93, 1946, 429457.

[12] J.A. Bloom and T.R. Reed, An Uncertainty Analysis of Some Real Functions for Image Processing Applications, Proceedings of the IEEE Intern. Conf. on Image Processing, 3, 1997, 670-673.

[13] H.G. Feichtinger and T. Strohmer, eds., Gabor Analysis and Algorithms. Theory and Applications, Birkhauser, 1997.

[14] A.S. Krylov and A.V. Vvedenskii, Software Package for Radial Distribution Function Calculation, J. of Non-Cryst. Solids, 192\&193, 1995, 683-687.

[15] A.N. Tikhonov and V.Y. Arsenin, Solution of Ill-posed Problems, V.H. Winston, Washington, DC, 1977.

[16] M. Abramowitz and I.A. Stegan, eds., Handbook of Mathematical Functions, McGraw-Hill, N.Y., 1969. 
[17] P.K. Suetin, Classical Orthogonal Polinomials, Nauka, Moscow, 1976 (in Russian).

[18] A.S. Krylov and A.V. Liakishev, An Inequality for the Norms of Hermite Functions on a Finite Interval, Vestnik Moskovskogo Universiteta, Seriya 15 - Vychislitel'naya Matematika i Kibernetika, No.1, 1999, 17-19 (in Russian).

[19] J. Max, Méthodes et Techniques de Traitement du Signal et Applications aux Mesures Physiques, Tome I, MASSON, 1981.

[20] N.V. Ershov, A.L. Ageev, A.V. Serikov, Regular Method in X-Ray Scattering Analysis of Amorphous and Liquid Metals, Phys. Stat. Sol., 121, 1984, 451-460.

[21] A.S. Krylov, J.F. Poliakoff and M.Stockenhuber, J. of Physics: Cond. Matter, to appear.

[22] A.S. Krylov and M.A. Spiridonov, Compact Presentation of Structure Dependencies of the Surface Layer of Liquid Copper, Rasplavi, N.4, 1993, 81-84 (in Russian). 


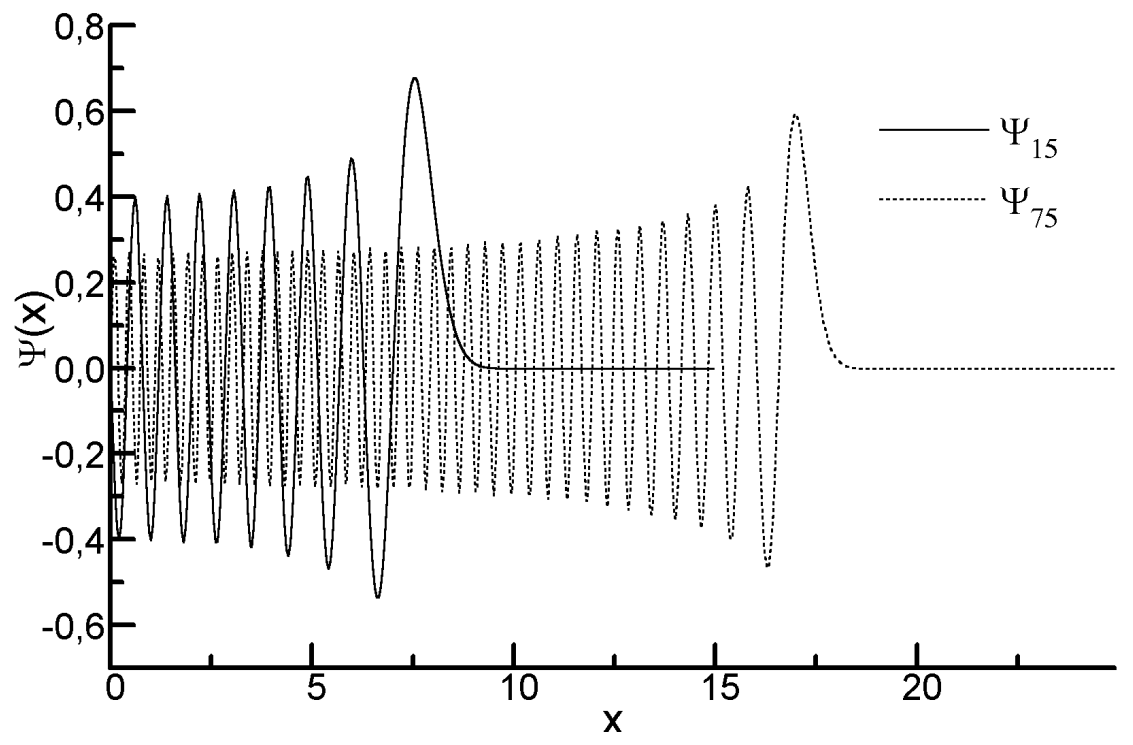

Figure 1: Odd Hermite functions

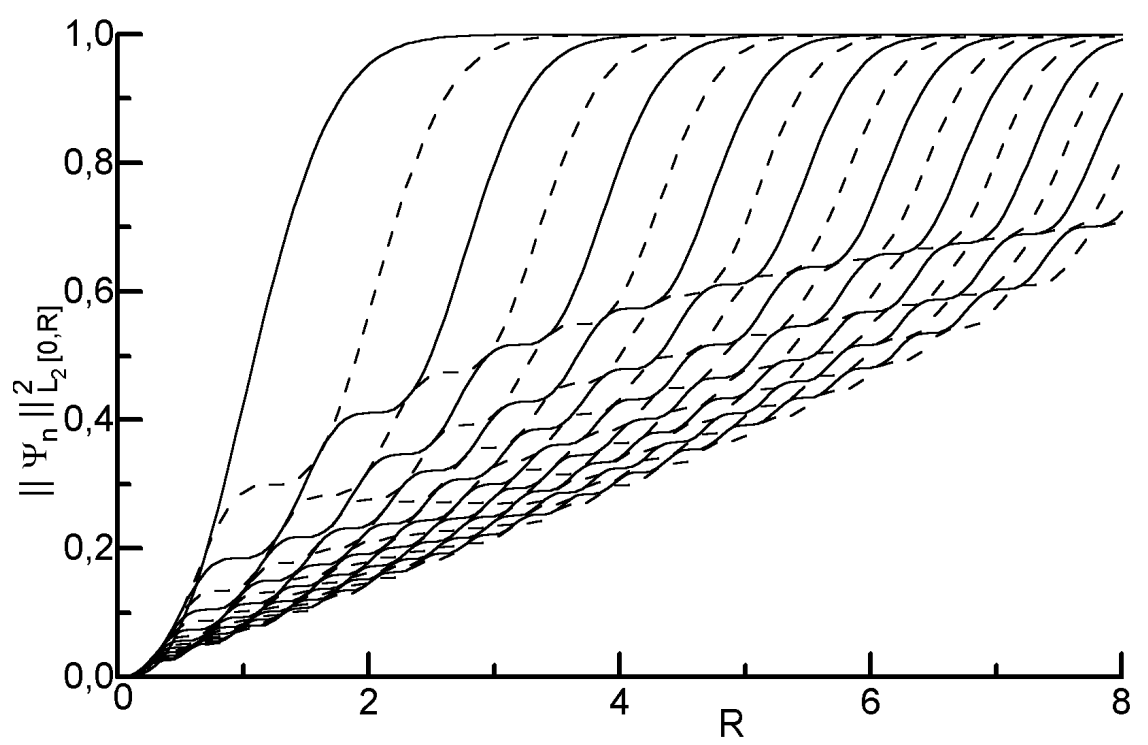

Figure 2: Norms of the first 20 odd Hermite functions in consequent order (from the left to the right) 


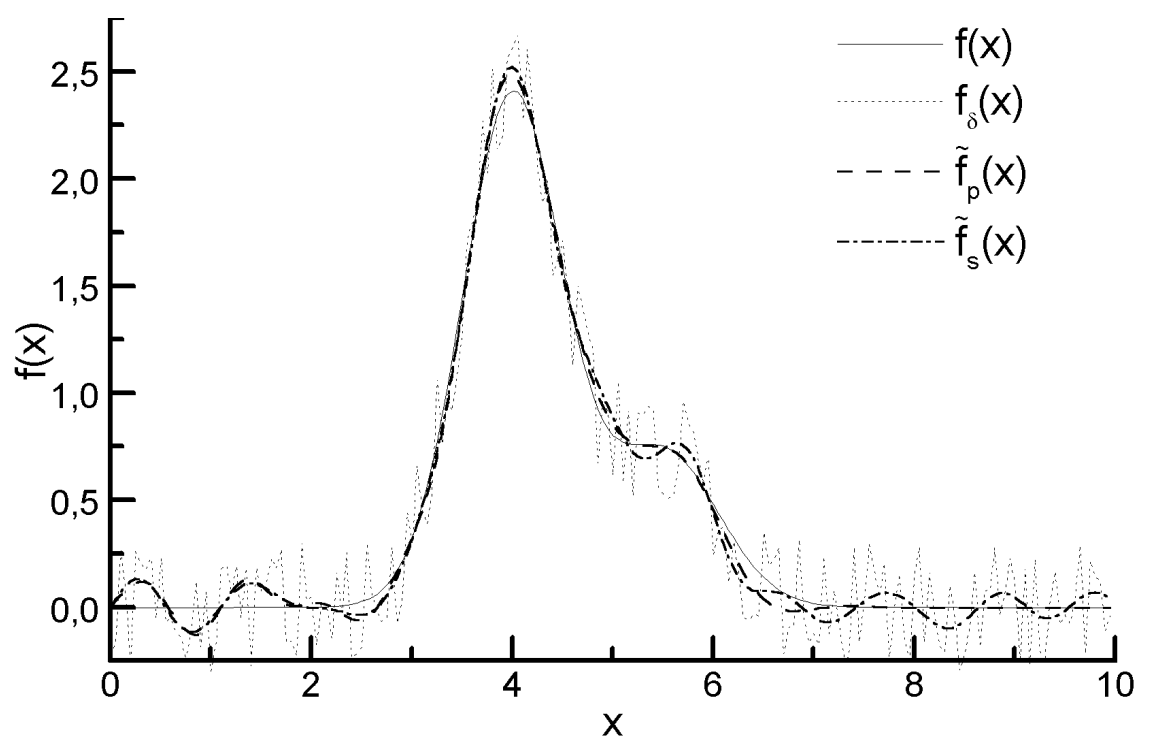

Figure 3: Sine Fourier filtering results

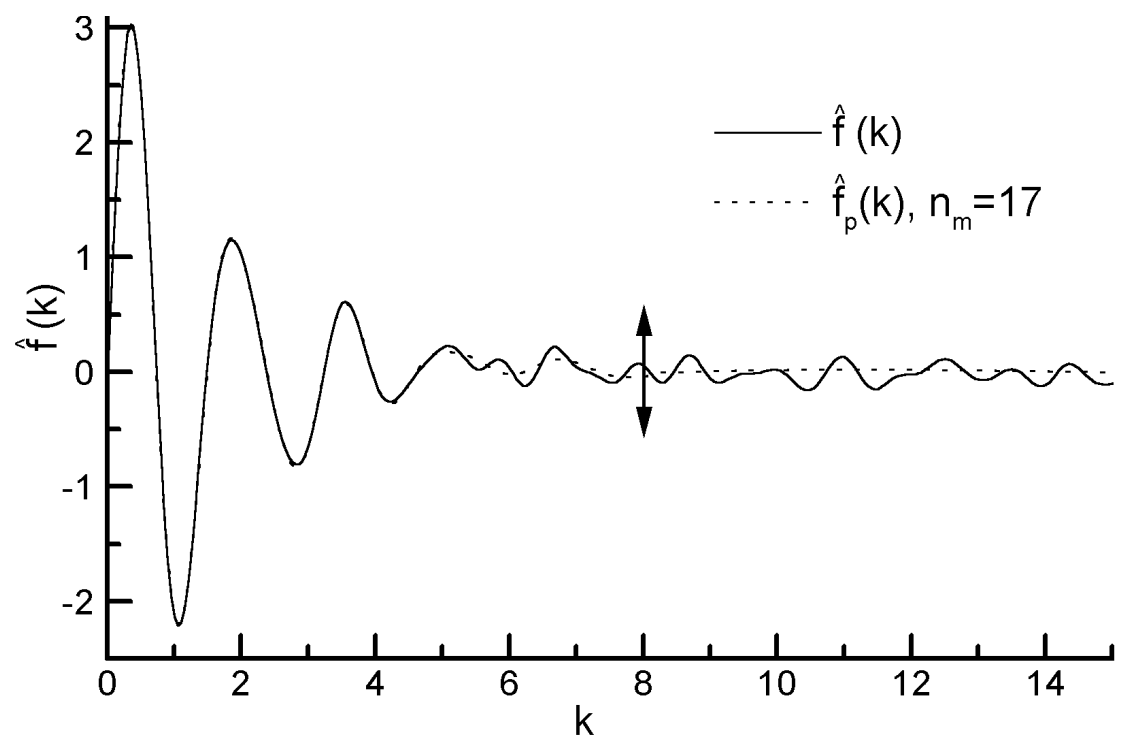

Figure 4: Sine Fourier filtering results 

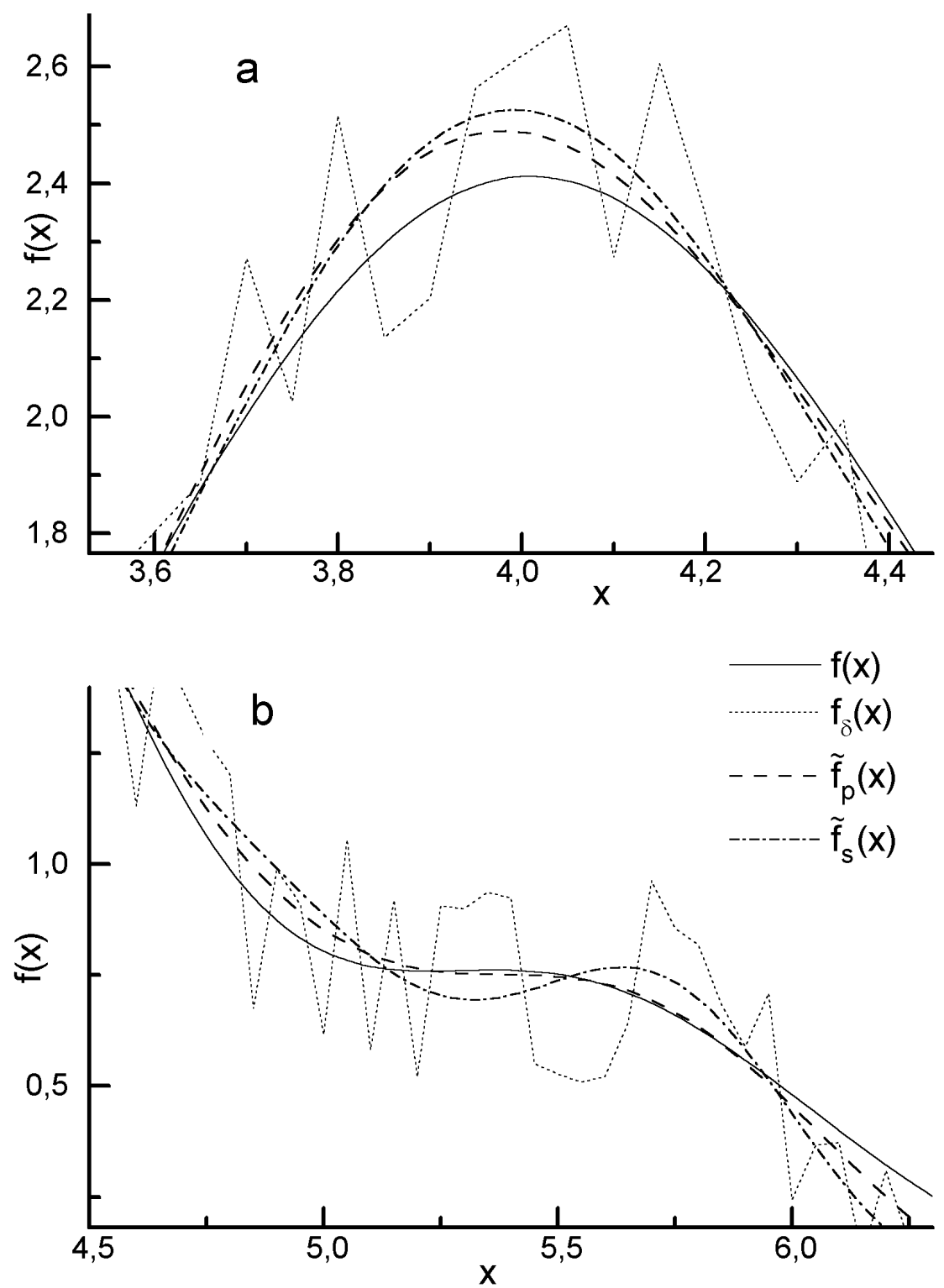

Figure 5: Details of Fig. 3 